RCNP-070

March 1994

\title{
Isomorphisms between Quantum Group Covariant q-Oscillator Systems Defined for $q$ and $q^{-1}$
}

\author{
N. Aizawa円 \\ Research Center for Nuclear Physics \\ Osaka University, Ibaraki, Osaka 567, Japan
}

\begin{abstract}
It is shown that there exists an isomorphism between q-oscillator systems covariant under $S U_{q}(n)$ and $S U_{q^{-1}}(n)$. By the isomorphism, the defining relations of $S U_{q^{-1}}(n)$ covariant q-oscillator system are transmuted into those of $S U_{q}(n)$. It is also shown that the similar isomorphism exists for the system of q-oscillators covariant under the quantum supergroup $S U_{q}(n / m)$. Furthermore the cases of q-deformed Lie (super)algebras constructed from covariant q-oscillator systems are considered. The isomorphisms between q-deformed Lie (super)algebras can not obtained by the direct generalization of the one for covariant q-oscillator systems.

PACS 02.20.Tw, 02.20.Qs
\end{abstract}

\footnotetext{
${ }^{1}$ Address after April 1994, Department of Applied Mathematics, Osaka Women's University, Sakai, Osaka 590, Japan
} 
Since the discovery of quantum deformation (the so-called q-deformation) of Lie groups and Lie algebras [1-5], many q-deformed objects have been introduced. We can mention q-deformed hyperplane [6], differential forms and derivatives on q-deformed hyperplane [7], q-(super)oscillators [8, 9], q-deformed covariant oscillator systems [10-13] and their generalization [14], q-symplecton [12, [15], reflection equation algebras [16], and so on. Almost all of these objects are essentially defined by the same algebraic structure, that is, Zamolodchikov-Faddeev algebra [17] or quantum group tensor [18]. However the relationship between q-deformed objects defined for different values of the deformation parameter $q$ is unclear. This problem has been discussed for the q-oscillator $H_{q}=\left\{a, a^{\dagger}, N\right\}$ and found that the central element of $H_{q}$ plays a crucial role. Assuming that the element $N$ and the central element are independent of $q$, Chaichian et al. derived the formula which transforms the elements of $H_{q_{1}}$ to the corresponding ones of $H_{q_{2}}$ [19]. Without such assumption, the present author found the one-to-one correspondence between the elements of $H_{q}$ and $H_{q^{-1}}$ which transmute the defining relations of $H_{q^{-1}}$ into those of $H_{q}$ [20]. The elements of $H_{q^{-1}}$ can be expressed in terms of those of $H_{q}$, therefore, we can say that the algebra $H_{q}$ is invariant under the replacement $q \leftrightarrow q^{-1}$. In mathematical language, $H_{q}$ is isomorphic to $H_{q^{-1}}$.

In this article, it is shown that there exists an isomorphism between q-oscillator systems which are covariant under $S U_{q}(n)$ and $S U_{q^{-1}}(n)$. By the isomorphism, the defining relations of $S U_{q^{-1}}(n)$ covariant q-oscillator system are transmuted into those of $S U_{q}(n)$. It is also shown that the similar isomorphism exists for the system of q-oscillators covariant under the quantum supergroup $S U_{q}(n / m)$. Furthermore q-deformed Lie (super)algebras constructed from covariant q-oscillator systems are considered. They are also covariant under the coaction of $S U_{q}(n)$ and $S U_{q}(n / m)$. It is shown that, unfortunately, the isomorphisms between covariant q-oscillator systems are not applicable to establish the isomorphisms between q-deformed Lie (super)algebras.

We start with the $S U_{q}(n)$ covariant q-oscillator system $\mathcal{A}_{q}$. It is generated by $2 n$ generators $\left\{A_{i}, A_{i}^{\dagger}, i=1, \cdots n\right\}$ and they satisfy the following defining relations [10, 11, 12]

$$
\begin{gathered}
A_{i} A_{j}=q A_{j} A_{i}, \quad A_{i}^{\dagger} A_{j}^{\dagger}=q^{-1} A_{j}^{\dagger} A_{i}^{\dagger}, \quad i<j \\
A_{i} A_{j}^{\dagger}=q A_{j}^{\dagger} A_{i}, \\
A_{i} A_{i}^{\dagger}-q^{2} A_{i}^{\dagger} A_{i}=1+\left(q^{2}-1\right) \sum_{k=1}^{i-1} A_{k}^{\dagger} A_{k} .
\end{gathered}
$$

Throughout this article, we assume to be $q \in \mathrm{R}, q>1$. The ${ }^{*}$-antiinvolution is introduced 
by

$$
\left(A_{i}\right)^{*}=A_{i}^{\dagger}, \quad\left(A_{i}^{\dagger}\right)^{*}=A_{i}
$$

The q-annihilation operators and the q-creation operators are covariant and contravariant tensors of rank 1 under the coaction of $S U_{q}(n)$, respectively. It means that the transformations

$$
\begin{aligned}
& A_{i} \rightarrow A_{i}^{\prime}=\sum_{k=1}^{n} t_{i j} A_{j}, \\
& A_{i}^{\dagger} \rightarrow A_{i}^{\dagger \prime}=\sum_{k=1}^{n} t_{i j}^{*} A_{j}^{\dagger},
\end{aligned}
$$

preserve the defining relations of $\mathcal{A}_{q}$ (11). Here we denote the generators of $S U_{q}(n)$ by $T=\left(t_{i j}\right)$ in matrix form and assume that $t_{i j}$ commute with all the generators of $\mathcal{A}_{q}$. The commutation relations of $t_{i j}$ are written using the R-matrix

$$
R T \otimes T=T \otimes T R
$$

the R-matrix is given by

$$
R=q \sum_{i=1}^{n} e_{i i} \otimes e_{i i}+\sum_{i \neq j} e_{i i} \otimes e_{j j}+\left(q-q^{-1}\right) \sum_{i>j} e_{i j} \otimes e_{j i}
$$

where $e_{i j}$ is the $n \times n$ matrix with entry 1 at position $(i, j)$ and 0 elsewhere. The $*_{-}$ antiinvolution is defined by

$$
T^{*}=\left(t_{j i}^{*}\right), \quad T^{*} T=T T^{*}=I,
$$

where $I$ is the $n \times n$ unit matrix. The quantum determinant, which is the central element of $G L_{q}(n)$, is defined by $\operatorname{det}_{q} T=\sum_{\sigma}(-q)^{l(\sigma)} t_{1 \sigma(1)} \cdots t_{n \sigma(n)}$, where $l(\sigma)$ is the minimal number of inversions in the permutation $\sigma$. We set $\operatorname{det}_{q} T=1$.

We also define the $S U_{q^{-1}}$ covariant q-oscillator system $\mathcal{A}_{q^{-1}}$ by replacing $q$ with $q^{-1}$. in (1). They form rank 1 tensors under the coaction of $S U_{q^{-1}}$. We denote q-deformed objects defined for $q^{-1}$ by attaching bar on their elements, e.g. $\mathcal{A}_{q^{-1}}=\left\{\bar{A}_{i}, \bar{A}_{i}^{\dagger}\right\}$. Our aim is to establish a relationship between $\mathcal{A}_{q}$ and $\mathcal{A}_{q^{-1}}$. It should be noted that the trivial relation $\bar{A}_{i}=A_{i}, \bar{A}^{\dagger}{ }_{i}=A_{i}^{\dagger}$ is prevented, since it concludes an unacceptable results ; $A_{i} A_{j}=0$, etc.

It is possible to relate the elements of $\mathcal{A}_{q}$ and $\mathcal{A}_{q^{-1}}$ so that the covariant q-oscillator system is invariant under $q \leftrightarrow q^{-1}$. We prove the following statement. 
There exists an isomorphism $\varphi: \mathcal{A}_{q} \rightarrow \mathcal{A}_{q^{-1}}$ such that $\varphi$ transmutes the defining relations of $\mathcal{A}_{q^{-1}}$ into those of $\mathcal{A}_{q}$. The explicit formulae are given by

$$
\bar{A}_{i}=\Gamma_{i-1}^{-1} \Gamma_{i}^{-1} A_{i}, \quad \bar{A}_{i}^{\dagger}=A_{i}^{\dagger} \Gamma_{i-1}^{-1} \Gamma_{i}^{-1}
$$

where

$$
\Gamma_{i} \equiv \sqrt{\left[A_{i}, A_{i}^{\dagger}\right]}, \quad \Gamma_{0} \equiv 1
$$

(Sketch of Proof)

Using the properties of $\Gamma_{i}$, we can prove the statement by direct calculations. Note that, using (11), $\Gamma_{i}(i \neq 0)$ is rewritten as

$$
\begin{aligned}
\Gamma_{i} & =\sqrt{A_{i+1} A_{i+1}^{\dagger}-q^{2} A_{i+1}^{\dagger} A_{i+1}} \\
& =\sqrt{1+\left(q^{2}-1\right) \sum_{k=1}^{i} A_{k}^{\dagger} A_{k} .}
\end{aligned}
$$

$\Gamma_{i}$ is not affected by the *antiinvolution $; \Gamma_{i}^{*}=\Gamma_{i}$. From these facts, we obtain the useful relations

$$
\begin{aligned}
{\left[\Gamma_{i}, \Gamma_{j}\right] } & =0, \\
A_{i} \Gamma_{j}=q \Gamma_{j} A_{i}, \quad A_{i}^{\dagger} \Gamma_{j} & =q^{-1} \Gamma_{j} A_{i}^{\dagger}, \quad i \leq j \\
{\left[A_{i}, \Gamma_{j}\right]=\left[A_{i}^{\dagger}, \Gamma_{j}\right] } & =0 . \quad i>j
\end{aligned}
$$

As an illustration, we take the last relation in (1)

$$
\bar{A}_{i} \bar{A}^{\dagger}{ }_{i}-q^{-2} \bar{A}_{i}^{\dagger} \bar{A}_{i}=1+\left(q^{-2}-1\right) \sum_{k=1}^{i-1} \bar{A}_{k}^{\dagger} \bar{A}_{k} .
$$

Substituting (6) into (10) and multiplying $\Gamma_{i-1} \Gamma_{i}$ from both left and right, we obtain

$$
A_{i} A_{i}^{\dagger}-A_{i}^{\dagger} A_{i}=\Gamma_{i-1}^{2} \Gamma_{i}^{2}\left\{1-\left(q^{2}-1\right) \sum_{k=1}^{i-1} \Gamma_{k-1}^{-2} \Gamma_{k}^{-2} A_{k}^{\dagger} A_{k}\right\} .
$$

Here the properties of $\Gamma_{i}$ (9) were used. Because of the identity

$$
\Gamma_{i}^{2}\left\{1-\left(q^{2}-1\right) \sum_{k=1}^{i} \Gamma_{k-1}^{-2} \Gamma_{k}^{-2} A_{k}^{\dagger} A_{k}\right\}=1,
$$

(11) reads

$$
\begin{aligned}
A_{i} A_{i}^{\dagger}-A_{i}^{\dagger} A_{i} & =\Gamma_{i}^{2} \\
& =1+\left(q^{2}-1\right) \sum_{k=1}^{i} A_{k}^{\dagger} A_{k} .
\end{aligned}
$$


Rearranging $A_{i}^{\dagger} A_{i}$, we obtain the last relation in (1).

The identity (12) is proved by mathematical induction. For $i=1$, the left hand side of (12) reads

$$
\begin{aligned}
& \Gamma_{1}^{2}\left\{1-\left(q^{2}-1\right) \Gamma_{1}^{-2} A_{1}^{\dagger} A_{1}\right\} \\
= & \Gamma_{1}^{2} \Gamma_{1}^{-2}\left\{\Gamma_{1}^{2}-\left(q^{2}-1\right) A_{1}^{\dagger} A_{1}\right\} \\
= & 1 .
\end{aligned}
$$

Assuming that (12) is valid for $\Gamma_{i}$, consider the case for $\Gamma_{i+1}$

$$
\begin{aligned}
& \Gamma_{i+1}^{2}\left\{1-\left(q^{2}-1\right) \sum_{k=1}^{i+1} \Gamma_{k-1}^{-2} \Gamma_{k}^{-2} A_{k}^{\dagger} A_{k}\right\} \\
= & \Gamma_{i+1}^{2}\left\{\Gamma_{i}^{-2}-\left(q^{2}-1\right) \Gamma_{i}^{-2} \Gamma_{i+1}^{-2} A_{i+1}^{\dagger} A_{i+1}\right\} \\
= & \Gamma_{i}^{-2}\left\{\Gamma_{i+1}^{2}-\left(q^{2}-1\right) A_{i+1}^{\dagger} A_{i+1}\right\} \\
= & \Gamma_{i}^{-2} \Gamma_{i}^{2}=1 .
\end{aligned}
$$

The identity (12) has been proved.

From (6), it is obvious that $\varphi$ is a one-to-one correspondence. In order to show that $\varphi$ is an isomorphism, let us consider $\varphi^{\prime}: \mathcal{A}_{q^{-1}} \rightarrow \mathcal{A}_{q}$ defined by

$$
A_{i}=\bar{\Gamma}_{i-1}^{-1} \bar{\Gamma}_{i}^{-1} \bar{A}_{i}, \quad A_{i}^{\dagger}=\bar{A}_{i}^{\dagger} \bar{\Gamma}_{i-1}^{-1} \bar{\Gamma}_{i}^{-1},
$$

and show that $\varphi \circ \varphi^{\prime}=\varphi^{\prime} \circ \varphi=1$. To this end, it is enough to note the relation

$$
\begin{aligned}
\bar{\Gamma}_{i} & =\sqrt{\left[\bar{A}_{i}, \bar{A}^{\dagger} i\right]} \\
& =\sqrt{\Gamma_{i-1}^{-2} \Gamma_{i}^{-2}\left(A_{i} A_{i}^{\dagger}-q^{2} A_{i}^{\dagger} A_{i}\right)} \\
& =\Gamma_{i}^{-1} .
\end{aligned}
$$

Therefore the statement has been proved.

It is emphasized that, in the limit of $q \rightarrow 1$, the both hand sides of (6) are reduced to the same bosonic oscillators.

Let us next consider the q-deformed Lie algebra $u_{q}(n)$ which is constructed from $\mathcal{A}_{q}$. As in the limit of $q \rightarrow 1$, the bilinear forms of q-creation and q-annihilation operators can define $u_{q}(n)$,

$$
E_{i j}=A_{i}^{\dagger} A_{j}, \quad E_{i j}^{*}=E_{j i}
$$


The commutation relations among $E_{i j}$ 's are obtained by using (1). In terms of the Rmatrix, (11) is rewritten as follows,

$$
\begin{aligned}
A_{j} A_{i} & =q^{-1} \sum_{k l} R_{i j, k l} A_{k} A_{l} \\
A_{j}^{\dagger} A_{i}^{\dagger} & =q^{-1} \sum_{k l} R_{k l, i j} A_{k}^{\dagger} A_{l}^{\dagger} \\
A_{j} A_{i}^{\dagger} & =\delta_{i j}+q \sum_{k l} R_{i l, k j} A_{k}^{\dagger} A_{l}
\end{aligned}
$$

The commutation relations of $u_{q}(n)$ are given by

$$
\begin{aligned}
& q \sum_{a b c d e f} R_{a b, \mu \nu}^{t_{1}} R_{a \rho, c d}^{-1} R_{c f, e \sigma} E_{e f} E_{d b} \\
- & q^{-1} \sum_{a b c d e f} R_{\mu a, b c} R_{c d, e \nu} R_{f e, \rho \sigma}^{t_{1}} E_{f d} E_{b a} \\
= & q \sum_{a b} R_{a b, \mu \nu}^{t_{1}} \delta_{\rho \sigma} E_{a b}-q^{-1} \sum_{a b} R_{a b, \rho \sigma}^{t_{1}} \delta_{\mu \nu} E_{a b} \\
- & q^{-1} \omega \sum_{a b c} R_{a b, \mu \nu}^{t_{1}} R_{c a, \rho \sigma}^{t_{1}} E_{c b}
\end{aligned}
$$

where $t_{1}$ means the transposition in the first space and $\omega \equiv q-q^{-1}$. This complicated relations are reduced to the usual commutation relations of $u(n)$ in the limit of $q \rightarrow 1$

$$
\left[E_{\mu \nu}, E_{\rho \sigma}\right]=\delta_{\nu \rho} E_{\mu \sigma}-\delta_{\mu \sigma} E_{\nu \rho}
$$

since the R-matrix is reduced to the unit matrix : $R_{i j, k l} \rightarrow \delta_{i k} \delta_{j l}$. From now on we adopt the equation (15) as the defining relation of $u_{q}(n)$ without the aid of covariant q-oscillator realization (13). The Hopf algebra structure for this $u_{q}(n)$ is still an open problem.

The algebra $u_{q}(n)$ forms the $S U_{q}(n)$ tensor of rank $(1,1)$, that is, the relation (15) is preserved by the transformation

$$
E_{i j} \quad \rightarrow \quad E_{i j}^{\prime}=\sum_{k l} t_{i k}^{*} t_{j l} E_{k l}
$$

This can be proved by direct calculation using the properties of the R-matrix i.e. (四), Yang-Baxter equation and $R_{i j, k l}=R_{l k, j i}$.

We expect that $u_{q^{-1}}(n)$ is isomorphic to $u_{q}(n)$ and the isomorphism is given by

$$
\begin{aligned}
\bar{E}_{i j} & =q^{3} \Gamma_{i-1}^{-1} \Gamma_{i}^{-1} \Gamma_{j-1}^{-1} \Gamma_{j}^{-1} E_{i j}, \quad i<j \\
\bar{E}_{i i} & =q^{2} \Gamma_{i-1}^{-2} \Gamma_{i}^{-2} E_{i i}
\end{aligned}
$$


where $\bar{E}_{i j}$ denotes the element of $u_{q^{-1}}(n)$. However it does not hold except the case of $n=2$. In order to show it, let us first consider the case of $n=2$. For $n=2$, (15) is reduced to

$$
\begin{aligned}
& {\left[E_{11}, E_{22}\right]=0} \\
& E_{11} E_{12}-q^{2} E_{12} E_{11}=E_{12}, \quad\left[E_{22}, E_{12}\right]=-E_{12}-\left(q^{2}-1\right) E_{12} E_{11}, \\
& q^{2} E_{12} E_{21}-E_{21} E_{12}=\left(q^{2}-1\right) E_{11}^{2}+E_{11}-E_{22}
\end{aligned}
$$

and $\Gamma_{i}(i=1,2)$ are restricted to the second expression in eq.(8)

$$
\Gamma_{1}=\sqrt{1+\left(q^{2}-1\right) E_{11}}, \quad \Gamma_{2}=\sqrt{1+\left(q^{2}-1\right)\left(E_{11}+E_{22}\right)} .
$$

It is easy to see that $E_{11}+E_{22}$ is a central element of this algebra, therefore, $\Gamma_{2}$ is also a central element. The non-trivial commutation relations are given by

$$
\Gamma_{1} E_{12}=q E_{12} \Gamma_{1}, \quad \Gamma_{2} E_{21}=q^{-1} E_{21} \Gamma_{1}
$$

It is proved by direct calculation that the isomorphism between $u_{q}(2)$ and $u_{q^{-1}}(2)$ is given by eq.(17). Here, we give only one example, the last relation of (19)

$$
q^{-2} \bar{E}_{12} \bar{E}_{21}-\bar{E}_{21} \bar{E}_{12}=\left(q^{-2}-1\right) \bar{E}_{11}^{2}+\bar{E}_{11}-\bar{E}_{22} \text {. }
$$

After substituting (17) into (21), we can arrange $\Gamma_{i}$ to the left of $E_{k l}$ by making use of (20)

$$
\Gamma_{1}^{-2} \Gamma_{2}^{-2}\left(q^{2} E_{12} E_{21}-E_{12} E_{21}\right)=\left(1-q^{2}\right) \Gamma_{1}^{-2} E_{11}^{2}+E_{11}-\Gamma_{2}^{-2} E_{22},
$$

where we dropped the common factor $\Gamma_{1}^{-2}$. Multiplying $\Gamma_{1}^{2} \Gamma_{2}^{2}$ from the left, we obtain the last equation of (19). Furthermore because of (17),

$$
\bar{\Gamma}_{1}=\Gamma_{1}^{-1}, \quad \bar{\Gamma}_{2}=\Gamma_{2}^{-1},
$$

hold. Therefore the isomorphism between $u_{q}(2)$ and $u_{q^{-1}}(2)$ has been proved.

On the other hand, for $n \geq 3, \sum_{i=1}^{n} E_{i i}$ is no longer a central element, so that the commutation relation between $\Gamma_{i}$ and $E_{k l}$ becomes quite complicated and the mechanism which makes $u_{q}(2)$ be isomorphic to $u_{q^{-1}}(2)$, namely arranging $\Gamma_{i}$ to the left of $E_{k l}$, does not work. Therefore eq.(17) does not give the isomorphism between $u_{q}(n)$ and $u_{q^{-1}}(n)$ for $n \geq 3$.

The isomorphisms discussed here can be generalized to the $S U_{q}(n / m)$ covariant qoscillator system $\mathcal{B}_{q}$ which is generated by $2 n$ even generators $\left\{A_{i}, A_{i}^{\dagger}, i=1,2, \cdots, n\right\}$ 
and $2 m$ odd generators $\left\{B_{r}, B_{r}^{\dagger}, r=1,2, \cdots, m\right\}$. The ${ }^{*}$-antiinvolution of a generator without dagger gives the corresponding one with dagger and vice versa. The $2(n+m)$ generators satisfy the following defining relations [11]

$$
\begin{aligned}
& A_{i} A_{j}=q A_{j} A_{i}, \quad i<j \\
& A_{i} A_{j}^{\dagger}=q A_{j}^{\dagger} A_{i}, \quad i \neq j \\
& A_{i} A_{i}^{\dagger}-q^{2} A_{i}^{\dagger} A_{i}=1+\left(q^{2}-1\right) \sum_{k=1}^{i-1} A_{k}^{\dagger} A_{k}, \\
& A_{i} B_{r}=q B_{r} A_{i}, \quad A_{i} B_{r}^{\dagger}=q B_{r}^{\dagger} A_{i}, \\
& B_{r} B_{s}=-q B_{s} B_{r}, \quad r<s \\
& B_{r} B_{s}^{\dagger}=-q B_{s}^{\dagger} B_{r}, \quad r \neq s \\
& B_{r} B_{r}^{\dagger}+B_{r}^{\dagger} B_{r}=1+\left(q^{2}-1\right) \sum_{k=1}^{n} A_{k}^{\dagger} A_{k}+\left(q^{2}-1\right) \sum_{s=1}^{r-1} B_{s}^{\dagger} B_{s}, \\
& B_{r}^{2}=\left(B_{r}^{\dagger}\right)^{2}=0,
\end{aligned}
$$

and their *-involution. The algebra $\mathcal{B}_{q}$ forms a rank 1 tensor of $S U_{q}(n / m)$. We assume that even generators $\mathcal{B}_{q}$ commute with all of $S U_{q}(n / m)$, odd generators of $\mathcal{B}_{q}$ commute with even ones of $S U_{q}(n / m)$, while they anticommute with odd ones of $S U_{q}(n / m)$. The coaction of $S U_{q}(n / m)$ on $\mathcal{B}_{q}$ is defined by

$$
\begin{aligned}
\alpha_{i} \quad \rightarrow \quad \alpha_{i}^{\prime} & =\sum_{j=1}^{n+m} t_{i j} \alpha_{j}, \\
\alpha_{i}^{\dagger} \quad \rightarrow \quad \alpha_{i}^{\dagger \prime} & =\sum_{j=1}^{n+m}(-)^{p\left(t_{i j}\right) p\left(\alpha_{j}\right)} t_{i j}^{*} \alpha_{j}^{\dagger},
\end{aligned}
$$

where $t_{i j} \in S U_{q}(n / m)$ and we introduced the unified notations for $\mathcal{B}_{q}$

$$
\alpha_{i}=A_{i} \quad \text { for } 1 \leq i \leq n, \quad \alpha_{i+n}=B_{i} \quad(1 \leq i \leq m) . \text { etc. }
$$

And $p(a)$ denotes the parity of operator $a$, namely $p(a)=1$ for odd $a, p(a)=0$ for even $a$.

The R-matrix for $S U_{q}(n / m)$ is given by 9]

$$
R=\sum_{i}^{n+m} q^{1-2 p(i)} e_{i i} \otimes e_{i i}+\sum_{i \neq j} e_{i i} \otimes e_{j j}+w \sum_{i>j} e_{i j} \otimes e_{j i}
$$

where $p(i)$ denotes the parity of $i$ th basis vector. We prove the following relationship between $\mathcal{B}_{q}$ and $\mathcal{B}_{q^{-1}}$. 
There exists an isomorphism $\varphi: \mathcal{B}_{q} \rightarrow \mathcal{B}_{q^{-1}}$ such that $\varphi$ transmutes the defining relations of $\mathcal{B}_{q^{-1}}$ into those of $\mathcal{B}_{q}$. The explicit formulae are given by

$$
\begin{aligned}
& \bar{A}_{i}=\Gamma_{i-1}^{-1} \Gamma_{i}^{-1} A_{i}, \quad \bar{A}_{i}^{\dagger}=A_{i}^{\dagger} \Gamma_{i-1}^{-1} \Gamma_{i}^{-1} \\
& \bar{B}_{r}=\Lambda_{r-1}^{-1} \Lambda_{r}^{-1} B_{r}, \quad \bar{B}_{r}^{\dagger}=B_{r}^{\dagger} \Lambda_{r-1}^{-1} \Lambda_{r}^{-1},
\end{aligned}
$$

where

$$
\begin{aligned}
\Gamma_{i} & \equiv \sqrt{\left[A_{i}, A_{i}^{\dagger}\right]}, \quad \Gamma_{0} \equiv 1, \\
\Lambda_{r} & \equiv \sqrt{B_{r} B_{r}^{\dagger}+q^{2} B_{r}^{\dagger} B_{r}}, \quad \Lambda_{0} \equiv \Gamma_{n} .
\end{aligned}
$$

(Sketch of Proof)

As in the case of $S U_{q}(n)$ covariant q-oscillator, the statement can be proved by direct calculations using the commutation relations among $\Gamma_{i}, \Lambda_{r}$ and the generators of $\mathcal{B}_{q}$. Because of the last relation of eq.(22), $\Lambda_{r}(r \neq 0)$ can be rewritten as

$$
\Lambda_{r}=\left\{1+\left(q^{2}-1\right) \sum_{k=1}^{n} A_{k}^{\dagger} A_{k}+\left(q^{2}-1\right) \sum_{s=1}^{r} B_{s}^{\dagger} B_{s}\right\}^{1 / 2} .
$$

The ${ }^{*}$-antiinvolution does not change $\Lambda_{r} ; \Lambda_{r}^{*}=\Lambda_{r}$. Following useful relations can be shown easily

$$
\begin{aligned}
& {\left[\Gamma_{i}, \Lambda_{r}\right]=\left[\Lambda_{r}, \Lambda_{s}\right]=0,} \\
& {\left[B_{r}, \Gamma_{i}\right]=\left[B_{r}^{\dagger}, \Gamma_{i}\right]=0,} \\
& A_{i} \Lambda_{r}=q \Lambda_{r} A_{i}, \quad A_{i}^{\dagger} \Lambda_{r}=q^{-1} \Lambda_{r} A_{i}^{\dagger}, \\
& {\left[B_{r}, \Lambda_{s}\right]=\left[B_{r}^{\dagger}, \Lambda_{s}\right]=0, \quad \text { for } s<r} \\
& B_{r} \Lambda_{s}=q \Lambda_{s} B_{r}, \quad B_{r}^{\dagger} \Lambda_{s}=q^{-1} \Lambda_{s} B_{r}^{\dagger}, \quad \text { for } s \geq r
\end{aligned}
$$

It is not difficult to prove the statement using these relations together with eq.(9). As a showcase, we consider the last relation of eq.(22). Again, we denote the generators of $\mathcal{B}_{q^{-1}}$ by the operators with bar.

$$
\bar{B}_{r} \bar{B}_{r}^{\dagger}+\bar{B}_{r}^{\dagger} \bar{B}_{r}=1+\left(q^{-2}-1\right) \sum_{k=1}^{n} \bar{A}_{k}^{\dagger} \bar{A}_{k}+\left(q^{-2}-1\right) \sum_{s=1}^{r-1} \bar{B}_{s}^{\dagger} \bar{B}_{s} .
$$

We substitute (25) into (29), then using (9) and (28) we obtain

$$
\begin{aligned}
& B_{r} B_{r}^{\dagger}+q^{2} B_{r}^{\dagger} B_{r} \\
= & \Lambda_{r-1}^{2} \Lambda_{r}^{2}\left\{1-\left(q^{2}-1\right) \sum_{k=1}^{n} \Gamma_{k-1}^{-2} \Gamma_{k}^{-2} A_{k}^{\dagger} A_{k}-\left(q^{2}-1\right) \sum_{s=1}^{r-1} \Lambda_{s-1}^{-2} \Lambda_{s}^{-2} B_{s}^{\dagger} B_{s}\right\} \\
= & \Lambda_{r-1}^{2} \Lambda_{r}^{2}\left\{\Gamma_{n}^{-2}-\left(q^{2}-1\right) \sum_{s=1}^{r-1} \Lambda_{s-1}^{-2} \Lambda_{s}^{-2} B_{s}^{\dagger} B_{s}\right\} .
\end{aligned}
$$


The relation (12) was used to derive the last line. As is shown later, an analogous identity to (12) holds

$$
\Lambda_{r}^{2}\left\{\Gamma_{n}^{-2}-\left(q^{2}-1\right) \sum_{s=1}^{r} \Lambda_{s-1}^{-2} \Lambda_{s}^{-2} B_{s}^{\dagger} B_{s}\right\}=1 .
$$

Because of this identity, (30) can be rewritten

$$
\begin{aligned}
& B_{r} B_{r}^{\dagger}+q^{2} B_{r}^{\dagger} B_{r} \\
= & \Lambda_{r}^{2} \\
= & 1+\left(q^{2}-1\right) \sum_{k=1}^{n} A_{k}^{\dagger} A_{k}+\left(q^{2}-1\right) \sum_{s=1}^{r} B_{s}^{\dagger} B_{s} .
\end{aligned}
$$

Rearranging $B_{r}^{\dagger} B_{r}$, we obtain the last relation of (22).

The identity (31) is proved by mathematical induction. For $r=1$, the left hand side of (31) reads

$$
\begin{aligned}
& \Lambda_{1}^{2}\left\{\Gamma_{n}^{-2}-\left(q^{2}-1\right) \Lambda_{1}^{-2} \Lambda_{0}^{-2} B_{1}^{\dagger} B_{1}\right\} \\
= & \Gamma_{n}^{-2}\left\{\Lambda_{1}^{2}-\left(q^{2}-1\right) B_{1}^{\dagger} B_{1}\right\}
\end{aligned}
$$

By definition of $\Lambda_{1}$, it is obviously reduced to unity. Assuming that (31) is valid for $\Lambda_{r}$, consider the case of $\Lambda_{r+1}$

$$
\begin{aligned}
& \Lambda_{r+1}^{2}\left\{\Gamma_{n}^{-2}-\left(q^{2}-1\right) \sum_{s=1}^{r+1} \Lambda_{s-1}^{-2} \Lambda_{s}^{-2} B_{s}^{\dagger} B_{s}\right\} \\
= & \Lambda_{r+1}^{2}\left\{\Lambda_{r}^{-2}-\left(q^{2}-1\right) \Lambda_{r}^{-2} \Lambda_{r+1}^{-2} B_{r+1}^{\dagger} B_{r+1}\right\} \\
= & \Lambda_{r}^{-2}\left\{\Lambda_{r+1}^{2}-\left(q^{2}-1\right) B_{r+1}^{\dagger} B_{r+1}\right\} \\
= & 1 .
\end{aligned}
$$

Therefore the identity (31) has been proved.

It can be easily seen that the map $\varphi^{\prime}: \mathcal{B}_{q^{-1}} \rightarrow \mathcal{B}_{q}$ defined by

$$
\begin{aligned}
& A_{i}=\bar{\Gamma}_{i-1}^{-1} \bar{\Gamma}_{i}^{-1} \bar{A}_{i}, \quad A_{i}^{\dagger}=\bar{A}_{i}^{\dagger} \bar{\Gamma}_{i-1}^{-1} \bar{\Gamma}_{i}^{-1} \\
& B_{r}=\bar{\Lambda}_{r-1}^{-1} \bar{\Lambda}_{r}^{-1} \bar{B}_{r}, \quad B_{r}^{\dagger}=\bar{B}_{r}^{\dagger} \bar{\Lambda}_{r-1}^{-1} \bar{\Lambda}_{r}^{-1},
\end{aligned}
$$

is the inverse map of $\varphi$, because of the relation

$$
\bar{\Lambda}_{r}=\Lambda_{r}^{-1} .
$$

This completes the proof of the statement. 
It is natural to extend the isomorphism to the q-deformed Lie superalgebra $u_{q}(n / m)$ constructed from $\mathcal{B}_{q}$. As in the case of $u_{q}(n)$, trivial extension is not valid against our expectation. Let us show it in the simplest case $u_{q}(1 / 1)$. [11] The generators of $u_{q}(1 / 1)$ are constructed by

$$
Q=A^{\dagger} B, \quad Q^{\dagger}=B^{\dagger} A, \quad X=A^{\dagger} A, \quad Y=B^{\dagger} B .
$$

They satisfy the following commutation relations

$$
\begin{aligned}
& Q^{2}=0, \\
& q^{2} Q Q^{\dagger}+q^{-2} Q^{\dagger} Q=X+q^{-2} Y+\left(q^{2}-1\right) X^{2}, \\
& X Q-q^{2} Q X=Q, \\
& Y Q=0, \quad q^{2} Q Y=Q+\left(q^{2}-1\right) X Q, \\
& {[X, Y]=0,}
\end{aligned}
$$

and their *-involution. We regard (35) as the defining relations of $u_{q}(1 / 1)$ without the aid of (34). According to (34), we expect that $u_{q^{-1}}$ is isomorphic to $u_{q}(1 / 1)$ and the isomorphism is given by

$$
\begin{aligned}
& \bar{Q}=q^{3} \Gamma^{-2} \Lambda^{-1} Q, \quad \bar{Q}^{\dagger}=q^{3} Q^{\dagger} \Gamma^{-2} \Lambda^{-1}, \\
& \bar{X}=q^{2} \Gamma^{-2} X, \quad \bar{Y}=q^{2} \Gamma^{-2} \Lambda^{-2} Y,
\end{aligned}
$$

where

$$
\Gamma=\sqrt{1+\left(q^{2}-1\right) X}, \quad \Lambda=\sqrt{1+\left(q^{2}-1\right)(X+Y)} .
$$

As an example, we consider the second equation of (35)

$$
q^{-2} \bar{Q} \bar{Q}^{\dagger}+q^{2} \bar{Q}^{\dagger} \bar{Q}=\bar{X}+q^{2} \bar{Y}+\left(q^{-2}-1\right) \bar{X}^{2} .
$$

Substituting (36) into this equation and multiplying $q^{-2} \Gamma^{2} \Lambda^{2}$ from the left, we obtain

$$
q^{2}\left(Q Q^{\dagger}+Q^{\dagger} Q\right)=X+\left(q^{2}-1\right) X^{2}+q^{2} Y+\left(q^{4}-1\right) X Y .
$$

The correct equation can not be derived unless the relation

$$
q^{2} X Y+Y-Q^{\dagger} Q=0,
$$

holds. However eq.(38) does not hold without the aid of the covariant q-oscillator realization (34). Therefore we have shown that (36) does not give the isomorphism between $u_{q}(1 / 1)$ and $u_{q^{-1}}(1 / 1)$. 
In this article, we have shown that, in the case of $S U_{q}(n)$ and $S U_{q}(n / m)$, the covariant q-oscillator systems defined for $q$ are isomorphic to the ones for $q^{-1}$. The final goal of investigation along the line presented here is to establish relationships between all kinds of q-deformed objects defined for $q$ and $q^{-1}$. This is not a easy but a challenging problem. As has seen in the case of q-deformed Lie algebra, the established isomorphism between covariant q-oscillators can not be generalized directly to other q-deformed objects. For the q-deformed Lie algebras, we will have to reanalyze the isomorphism based on the structure of the algebra itself without the aid of covariant q-oscillator realizations. We can mention an another example, namely extended covariant q-oscillator system. It is an algebra which consist of some copies of a covariant q-oscillator system. The mutual relationships among various copies should also be covariant under the quantum group coaction. This requirement concludes that generators of a covariant q-oscillator system do not commute with their copies, and the commutation relations among various copies becomes non-trivial. For example, commutation relations between $S U_{q}(n)$ covariant qoscillator system $\mathcal{A}_{q}=\left\{A_{i}, A_{i}^{\dagger}\right\}$ and its copy $\left\{D_{i}, D_{i}^{\dagger}\right\}$ are given, in terms of the R-matrix, by [12]

$$
\begin{aligned}
D_{j} A_{i} & =q \sum_{k l} R_{i j, k l} A_{k} D_{l}, & A_{j}^{\dagger} D_{i}^{\dagger} & =q \sum_{k l} R_{k l, i j} D_{k}^{\dagger} A_{l}^{\dagger}, \\
A_{j} D_{i}^{\dagger} & =q \sum_{k l} R_{i l, k j} D_{k}^{\dagger} A_{l}, & D_{j} A_{i}^{\dagger} & =q \sum_{k l} R_{i l, k j} A_{k}^{\dagger} D_{l} .
\end{aligned}
$$

It can be easily verified that (6) does not give the isomorphism between this extended

$\mathcal{A}_{q}$ and the one defined for $q^{-1}$, although we do not give the proof here. This is due to the additional structure given by (39), we have to take it into consideration if we wish to establish the isomorphism.

One of the most important problem concerning the isomorphism discussed here is the relationships between quantum groups defined for $q$ and $q^{-1}$, e.g. $S U_{q}(n)$ and $S U_{q^{-1}}(n)$. Unfortunately, the result of the present article seems not to be applicable to the problem. It will be a future work.

\section{References}

[1] V. G. Drinfeld, Quantum Groups, ICM proceedings vol. 1, p798, New York: Berkeley (1986), (The American Mathematical Society 1987).

[2] M. Jimbo, Lett. Math. Phys. 10 (1985) 63 ; 11 (1986) 247 ; Comm. Math. Phys. $102(1986) 537$. 
[3] E. K. Sklyanin, Funct. Anal. Appl. 16 (1982) 263.

P.P. Kulish and N. Yu. Reshetikhin, J. Soviet. Math. 23 (1983) 2435.

[4] L. D. Faddeev, N. Yu. Reshetikhin and L. A. Takhtajan, Algebra Anal. 1 (1987) 178.

[5] S. L. Woronowicz, Publ. RIMS. Kyoto univ. 23 (1987) 117 ; Comm. Math. Phys. $111(1987) 613 ; \mathbf{1 2 2}(1989) 125$.

[6] Yu. I. Manin, Quantum gruops and non-commutative geometry, Les publications du Centre de Recherches Mathématiques, Univ. Montréal (1988) ; Comm. Math. Phys. 123 (1989) 163.

[7] J. Wess and B. Zumino, Nucl. Phys. B (Proc. Suppl.) 18B (1990) 302.

[8] L. C. Biedenharn, J. Phys. A22 (1989) L873.

A. J. Macfarlane, J. Phys. A22 (1989) 4581.

C.-P. Sun and H.-C. Fu, J. Phys. A22 (1989) L983.

T. Hayashi, Comm. Math. Phys. 127 (1990) 129.

[9] M. Chaichian and P. P. Kulish, Phys. Lett. B234 (1990) 72.

[10] W. Pusz and S. L. Woronowicz, Rep. Math. Phys. 27 (1989) 231.

[11] M. Chaichian, P. P. Kulish and J. Lukierski, Phys. Lett. 262 (1991) 43.

[12] M. Nomura, J. Phys. Soc. Japan 60 (1991) 3260, 4060.

[13] C. Quesne, in Proceedings of 3rd International Wigner Symposium (to be published).

[14] J. Van der Jeugt, J. Phys. A26 (1993) L405.

[15] M. Nomura and L. C. Biedenharn, J. Math. Phys. 33 (1992) 3636.

[16] P. P. Kulish and E. K. Sklyanin, J. Phys. A25 (1992) 5963.

[17] P. P. Kulish, Phys. Lett. A161 (1991) 50.

[18] P. P. Kulish and R. Sasaki, Prog. Theore. Phys. 89 (1993) 741.

[19] M. Chaichian, M. N. Mnatsakanova and Yu. S. Vernov, Univ. of Helsinki preprint, HU-TFT-93-6.

[20] N. Aizawa, J. Phys. A26 (1993) 1115. 